\title{
Mechanical Vibration of Visco-Elastic Plate With Thickness Variation
}

\author{
Anupam Khanna, Ashish Kumar Sharma \\ Asst. Prof. Dept. of Mathematics MMEC, MMU (MULLANA), AMBALA, \\ INDIA \\ anupam_rajie@yahoo.co.in \\ Asst. Prof. Dept. of Mathematics, MANAV BHARTI UNIVERSITY, SOLAN \\ (H.P), INDIA. \\ ashishk482@gmail.com
}

\begin{abstract}
A mathematical study or model is constructed with an aim to assist the design engineers for the making of various structures used in the satellite and aeronautical engineering. Visco-elastic plates are being increasingly used in the aeronautical and aerospace industry as well as in other fields of modern technology. To use them a good understanding of their structural and dynamical behavior is needed. In the modern technology, the plates of variable thickness are widely used in engineering applications. A mathematical model is presented for the use of engineers and research workers in space technology; have to operate under elevated temperatures. Rayleigh Ritz approach is applied for the solution of the problem. Fundamental frequencies and deflection functions are calculated for first mode of vibration of a clamped plate with diverse values of thermal gradient and taper constants.
\end{abstract}

Keywords: Visco-elastic, Square plate, Parabolically, Thermal gradient, Taper constant.

\section{Introduction}

Vibration effects have always been a principle concern of engineers. In the epoch of science and technologies it is desired to design large machines with smooth operation and unwanted vibrations. Sometimes unwanted vibration causes fatigues. Unwanted vibration can damage electronic components of aerospace 
system, damage buildings by earthquake, bring tsunami, and contribute to toppling of tall smokestacks, collapse of a suspension bridge in a windstorm. There are a multitude of applications where vibration effect is required e. g. in string and percussion instruments, in the design of loudspeakers, space shuttles, satellites where discrepancies in the temperature also affects the vibration effect. Controlled vibration effects are also required in health industry, paper industry, design of structures, building construction, reducing soil adhesion and many more areas engross vibration upshot.

Hence vibrations totally affect our day-to-day life. Thus for design engineers and scientist, it has always been a necessity to optimize or to control the effect of unwanted vibrations as much as possible. Present work is a full-fleshed endeavor to assist the design officers, industry people to come up to the situation.

In the recent past, there has been increasingly great interest in high strength, corrosion resistance and high temperature performance materials for structural components used in mechanical, aerospace, ocean engineering, electronic and optical equipments. Modern engineering structures are based on different types of design, which involve various types of anisotropic and non-homogeneous materials in the form of their structure components. Depending upon the requirement, durability and reliability, materials are being developed so that they can be used to give better strength and efficiency. The equipment used in air-jet, communications and in other similar technological industries take into consideration such materials, which not only reduce the weight and size but also are reliable in terms of efficiency, strength and economy.

Recently, Leissa [1,2] has given the solution for rectangular plate of variable thickness. Kishor and Rao [3] have discussed non linear vibration of rectangular plate on visco-elastic foundation. Gupta, Johri and Vats [4] have discussed the thermal effect on vibration of non-homogeneous orthotropic rectangular plate having bi-directional parabolically varying thickness. Gupta and Khanna [5] have solved the problem of free vibration of visco-elastic rectangular plate with linearly thickness variations in both directions. Singh and Saxena [6] have discussed the transverse vibration of rectangular plate with bi-directional thickness variation. Sobotka [7] has investigated the vibration of rectangular orthotropic visco-elastic plates. Lal [8] studied transverse vibrations of orthotropic non-uniform rectangular plates with continuously varying density. Warade and Deshmukh [9] discussed thermal deflection of a thin clamped circular plate due to partially distributive heat supply. Sobotka [10] discussed rheology of orthotropic viscoelastic plates. Gupta and Kumar [12] analyzed vibration of non-homogeneous visco-elastic rectangular plates with linearly varying thickness. Hewitt [13] have considered vibration of triangular viscoelastic plates. Huffington and Hoppmann [14] have solved the problem of the transverse vibrations of rectangular orthotropic plates. Recently, Gupta and Kumar [15] study the effect of thermal gradient on free vibration of non-homogeneous visco elastic rectangular plate of parabolically varying thickness. 
The aim is to study two dimensional thermal effects on the vibration of viscoelastic square plate whose thickness varies linearly in $\mathrm{x}$-direction and temperature varies bi-parabolically in another direction. It is assumed that the plate is clamped on all the four edges and its temperature varies linearly in both the directions. Due to temperature variation, we assume that non homogeneity occurs in Modulus of Elasticity. For various numerical values of thermal gradient and taper constants; frequency for the first two modes of vibration are calculated with the help of latest software and all the results are shown in Graphs.

\section{Methodology}

Let the plate is subjected to a study two dimensional parabolically temperature distribution [2] i.e.

$$
T=T_{0}\left(1-x^{2} / a^{2}\right)\left(1-y^{2} / a^{2}\right)
$$

where, $T$ denotes the temperature excess above the reference temperature at any point on the plate and $T_{0}$ denotes the temperature at any point on the boundary of plate and " $a$ " is the length of a side of square plate. The temperature dependence of the modulus of elasticity for most of engineering materials can be expressed in this

$$
E=E_{0}(1-\gamma \tau)
$$

Where, $\mathrm{E}_{0}$ is the value of the Young's modulus at reference temperature i.e. $T=0$ and $\gamma$ is the slope of the variation of $\mathrm{E}$ with $T$. The modulus variation (2) become

$$
E=E_{0}\left[1-\alpha\left(1-x^{2} / a^{2}\right)\left(1-y^{2} / a^{2}\right)\right]
$$

where, $\alpha=\gamma T_{0}(0 \leq \alpha<1)$ thermal gradient.

Also, It is assumed that thickness also varies linearly in $\mathrm{x}$ - directions as shown below:

$$
h=h_{0}\left(1+\beta_{1} x / a\right)
$$

where, $\beta_{1}$ is taper parameters in $\mathrm{x}$ - directions respectively and $\mathrm{h}=\mathrm{h}_{0}$ at $\mathrm{x}=\mathrm{y}=0$.

The governing differential equation of transverse motion for visco-elastic square plate of variable thickness in Cartesian coordinate is [1]:

$$
\begin{aligned}
& {\left[\mathrm{D}_{1}\left(\mathrm{~W},{ }_{\mathrm{xxxx}}+2 \mathrm{~W},_{\mathrm{xxyy}}+\mathrm{W},,_{\mathrm{yyyy}}\right)+2 \mathrm{D}_{1},_{\mathrm{x}}\left(\mathrm{W},_{\mathrm{xxx}}+\mathrm{W},_{\mathrm{xyy}}\right)+2 \mathrm{D}_{1, \mathrm{y}}\left(\mathrm{W},{ }_{\mathrm{yyy}}+\mathrm{W},{ }_{\mathrm{yxx}}\right)+\right.} \\
& \left.\mathrm{D}_{1, \mathrm{xx}}\left(\mathrm{W},_{\mathrm{xx}}+v \mathrm{~W}_{\mathrm{yy}}\right)+\mathrm{D}_{1, \mathrm{yy}}\left(\mathrm{W},_{\mathrm{yy}}+v \mathrm{~W},_{\mathrm{xx}}\right)+2(1-v) \mathrm{D}_{1, \mathrm{xy}} \mathrm{W},_{\mathrm{xy}}\right]-\rho \mathrm{hp}^{2} \mathrm{~W}=0
\end{aligned}
$$


A comma followed by a suffix denotes partial differential with respect to that variable.

Here, $D_{1}$ is the flexural rigidity of plate i.e.

$$
D_{1}=E h^{3} / 12\left(1-v^{2}\right)
$$

and deflection function for free transverse vibrations of the plate can be written as, in the form of Levy type solution [5]

(7)

$$
W=[(x / a)(y / a)(1-x / a)(1-y / a)]^{2}\left[A_{1}+A_{2}(x / a)(y / a)(1-x / a)(1-y / a)\right]
$$

Put the value of $E \& h$ from equation (3) \& (4) in the equation (6), one obtain

$$
D_{1}=\left[E_{0}\left[1-\alpha\left(1-x^{2} / a^{2}\right)\left(1-y^{2} / a^{2}\right)\right] h_{0}^{3}\left(1+\beta_{1} x / a\right)^{3}\right] / 12\left(1-v^{2}\right)
$$

Rayleigh-Ritz technique is applied to solve the frequency equation. In this method, one requires maximum strain energy must be equal to the maximum kinetic energy. So it is necessary for the problem under consideration that

$$
\delta\left(V^{*}-T^{*}\right)=\mathbf{O}
$$

for arbitrary variations of $\mathrm{W}$ satisfying relevant geometrical boundary conditions.

Since the plate is assumed as clamped at all the four edges, so the boundary conditions are

$$
\left.\begin{array}{l}
W=W,_{x}=0, x=0, a \\
W=W,_{y}=0, y=0, a
\end{array}\right\}
$$

Now assuming the non-dimensional variables as

$$
X=x / a, Y=y / a, \bar{W}=W / a, \bar{h}=h / a
$$

The kinetic energy $\mathrm{T}^{*}$ and strain energy $\mathrm{V}^{*}$ are [2]

$$
T^{*}=(1 / 2) \rho p^{2} \overline{h_{0}} a^{5} \int_{0}^{1} \int_{0}^{1}\left[\left(1+\beta_{1} X\right) \overline{W^{2}}\right] d Y d X
$$

and

$$
\begin{aligned}
& V^{*}=Q \int_{0}^{1} \int_{0}^{1}\left[1-\alpha\left(1-X^{2}\right)\left(1-Y^{2}\right)\right]\left(1+\beta_{1} X\right)^{3}\left\{\left(\bar{W}_{X X}\right)^{2}+\left(\bar{W}_{, Y Y}\right)^{2}\right. \\
& \left.+2 v \bar{W}_{, X X} \bar{W}_{, Y Y}+2(1-v)\left(\bar{W}_{X Y}\right)^{2}\right\} d Y d X
\end{aligned}
$$

where, $Q=E_{0} h_{0}^{3} a^{3} / 24\left(1-v^{2}\right)$ 
Using equations (12) \& (13) in equation (9), one get

$$
\left(V^{* * *}-\lambda^{2} T^{* * *}\right)=0
$$

where,

$$
\begin{aligned}
& V^{* *}=\int_{0}^{1} \int_{0}^{1}\left[1-\alpha\left(1-X^{2}\right)\left(1-Y^{2}\right)\right]\left(1+\beta_{1} X\right)^{3}\left\{\left(\bar{W}_{X X}\right)^{2}+\left(\bar{W}_{Y Y}\right)^{2}\right. \\
& \left.+2 v \bar{W},_{X X} \bar{W}_{, Y Y}+2(1-v)\left(\bar{W}_{X Y}\right)^{2}\right\} d Y d X
\end{aligned}
$$

and

$$
T^{* * *}=\int_{0}^{1} \int_{0}^{1}\left[\left(1+\beta_{1} X\right) \overline{W^{2}}\right] d Y d X
$$

Here, $\lambda^{2}=12 \rho\left(1-v^{2}\right) a^{2} / E_{0} h_{0}^{2}$ is a frequency parameter.

Equation (16) consists two unknown constants i.e. $\mathrm{A}_{1} \& \mathrm{~A}_{2}$ arising due to the substitution of $\mathrm{W}$. These two constants are to be determined as follows

$$
\partial\left(V^{* * *}-\lambda^{2} T^{* * *}\right) / \partial A_{n}=0 \quad, \mathrm{n}=1,2
$$

On simplifying (2.17), one gets

$$
b n_{1} A_{1}+b n_{2} A_{2}=0 \quad, \mathrm{n}=1,2
$$

where, $b_{1}, b_{2}(n=1,2)$ involve parametric constant and the frequency parameter.

For a non-trivial solution, the determinant of the coefficient of equation (18) must be zero. So one gets, the frequency equation as

$$
\left|\begin{array}{l}
b_{11} b_{12} \\
b_{21} b_{22}
\end{array}\right|=0
$$

With the help of equation (19), one can obtains a quadratic equation in $\lambda^{2}$ from which the two values of $\lambda^{2}$ can found. These two values represent the two modes of vibration of frequency i.e. $\lambda_{1}$ (Mode1) $\& \lambda_{2}$ (Mode2) for different values of taper constant and thermal gradient for a clamped plate.

\section{Result and Discussion}

All calculations are carried out with the help of latest Matrix Laboratory computer software. Computation has been done for frequency of visco-elastic square plate for different values of taper constants $\beta_{1}$ and thermal gradient $\alpha$, at different points for first two modes of vibrations have been calculated numerically. 
In Table and Fig I: - It is clearly seen that value of frequency decreases as value of thermal gradient increases from 0.0 to 1.0 for $\beta_{1}=0.4$ and $\beta_{1}=0.6$ for both modes of vibrations.

In Table and Fig II: - Also it is obvious to understand the increment in frequency as value of tapper constant $\beta_{1}$ from 0.0 to 1.0 for $\alpha=0.4$ and $\alpha=0.6$ for both modes of vibrations.

On the comparison of above discussion we have seen that frequency is decreases when we increase the value of thermal gradient from 0.0 to 1.0 for $\beta_{1}=0.4$ and $\beta_{1}=0.6$ for both modes of vibrations and frequency is increases when the value of tapper constant $\beta_{1}$ from 0.0 to 1.0 for $\alpha=0.4$ and $\alpha=0.6$ for both modes of vibration.

Table I: Frequency Vs Thermal gradient

\begin{tabular}{|c|c|r|r|r|}
\hline $\boldsymbol{\alpha}$ & \multicolumn{2}{|c|}{$\boldsymbol{\beta}_{\mathbf{1}}=\mathbf{0 . 4}$} & \multicolumn{2}{c|}{$\boldsymbol{\beta}_{\mathbf{1}}=\mathbf{0 . 6}$} \\
& Mode 1 & Mode 2 & \multicolumn{1}{c|}{ Mode 1 } & \multicolumn{1}{c|}{ Mode 2 } \\
\hline 0 & 162.18 & 41.57 & 174.41 & 44.72 \\
0.2 & 153.63 & 39.85 & 164.55 & 42.96 \\
0.4 & 144.58 & 38.04 & 154.06 & 41.19 \\
0.6 & 134.94 & 36.11 & 142.83 & 39.11 \\
0.8 & 124.57 & 34.03 & 130.66 & 36.94 \\
1 & 113.26 & 31.74 & 117.26 & 34.49 \\
\hline
\end{tabular}

Fig I:- Frequency Vs Thermal gradient

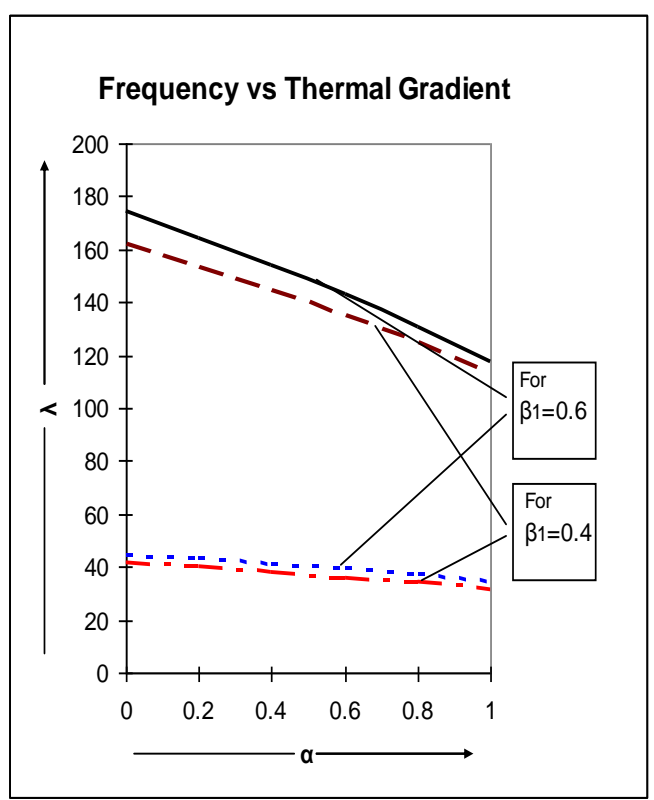


Table II: Frequency Vs Taper constant

\begin{tabular}{|c|c|c|c|c|}
\hline \multirow{2}{*}{$\beta_{\mathbf{1}}$} & \multicolumn{2}{|c|}{$\alpha=\mathbf{0 . 4}$} & \multicolumn{2}{c|}{$\alpha=\mathbf{0 . 6}$} \\
& Mode 1 & Mode 2 & Mode 1 & Mode 2 \\
\hline 0 & 125.71 & 32.53 & 117.37 & 30.63 \\
0.2 & 135.23 & 35.16 & 126.65 & 33.26 \\
0.4 & 144.61 & 38.12 & 134.94 & 36.11 \\
0.6 & 154.16 & 41.19 & 142.83 & 39.11 \\
0.8 & 163.86 & 44.28 & 150.69 & 42.21 \\
1 & 174.15 & 47.57 & 158.76 & 45.35 \\
\hline
\end{tabular}

Fig II:- Frequency Vs Tapper Constant

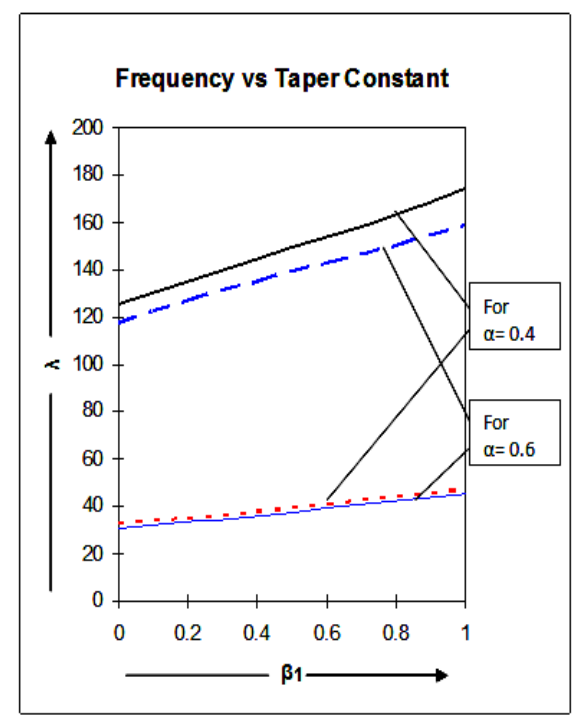

\section{Conclusion}

The objective of this paper is to clarify the characteristics of vibration of plates with variable thickness. Authors conclude that the results of present paper have a good convergence and satisfactory accuracy with available literature.

It is an approach to provide the guidelines (through theoretical models) for technocrats and design engineers so that they can analyze the models before finalizing any design of machine. 
Mechanical Vibration of.........

\section{ACKNOWLEDGEMENTS}

It is with a feeling of great pleasure that we would like to express my most sincere heartfelt gratitude to Chancellor of MANAV BHARTI UNIVERSITY, Solan (H.P, INDIA), Dr. RAJ KUMAR RANA and to my guide Dr. ANUPAM KHANNA, Asst. Prof. Dept. of Mathematics, MM University, Haryana (INDIA) for providing me the necessary facilities in the department and for giving me right way for research.

\section{References}

[1]. A.W.Leissa, Vibration of plate, NASA SP-160, 1969.

[2]. A.W.Leissa, Recent studies in plate vibration 1981-1985 part II, complicating effects, Shock and Vibration Dig., 19(1987), 10-24.

[3]. B.Kishor and J.S.Rao, Non linear vibration analysis of rectangular plate on a visco-elastic foundation, Aeronaut Quart. 39(1974), 37-46.

[4]. A.K.Gupta, T.Johri and R.P.Vats, "Thermal effect on vibration of nonhomogeneous orthotropic rectangular plate having bi-directional parabolically varying thickness", Proceeding of International Conference in World Congress on Engineering and Computer Science 2007, SanFrancisco, USA, 24-26 Oct, 2007, 784-787.

[5]. A.K.Gupta and A.Khanna, Vibration of visco-elastic rectangular plate with linearly thickness variations in both directions, Journal of Sound and Vibration ,301(2007), 450-457.

[6]. B.Singh and V.Saxena, Transverse vibration of rectangular plate with bidirectional thickness variation, Journal of Sound and Vibration 198(1996), 51-65.

[7]. Z. Sobotka, Vibration of rectangular orthotropic visco-elastic plate, Acta Techanica CSAV, 1969, 511-514.

[8]. R.Lal, Transverse vibrations of orthotropic non-uniform rectangular plates with continuously varying density, Indian J. Pure Appl. Math., 34(2003), 587-606.

[9]. R.W.Warade and K.C.Deshmukh, Thermal deflection of a thin clamped circular plate due to a partially distributive heat supply, Ganita, 55(2004), 179-186.

[10]. Z.Sobotka, Rheology of orthotropic visco-elastic plates, Proc. of 5th International Congress on Rheology, Univ. of Tokyo Press, Tokyo Univ., Park Press, Baltimore, 175-184, 1971.

[11]. Z.Sobotka, Free vibration of visco-elastic orthotropic rectangular plates, Acta Techanica CSAV, 1978, 678-705.

[12]. A.K.Gupta and L.Kumar, Thermal effect on vibration of non-homogenous visco-elastic rectangular plate of linear varying thickness, Meccanica, 43(2008), 47-54.

[13]. J.S.Hewitt, Vibration of a triangular visco-elastic plate, J. Engg. Mech. Div., EM6, 100(1974), 1143-1148. 
[14]. A.K.Gupta and L.Kumar, Effect of thermal gradient on free vibration of non-homogeneous visco-elastic rectangular plate of parabolically varying thickness, Acta Technica CSAV, 54(2009), 359-374.

[15]. Anupam Khanna, Ashish Kumar Sharma, Vibration Analysis of ViscoElastic Square Plate of Variable Thickness with Thermal Gradient, International Journal of Engineering and Applied Sciences, Turkey, Vol. 3 - Issue 4, pp. 1-6, 2011. 